\title{
ON COMMUTATIVITY THEOREMS FOR RINGS
}

\author{
H.A.S. ABUJABAL and M.S. KHAN \\ Department of Mathematics \\ Faculty of Science \\ King Abdul Aziz University \\ P.0. BOX 9028, Jeddah - 21413 \\ Saudi Arabia
}

(Received February 2, 1989)

ABSTRACT. Let $R$ be an associative ring with unity. It is proved that if $R$ satisfies the polynomial identity $\left[x^{n} y-y^{m} x^{n}, x\right]=0(m>1, n>1)$, then $R$ is commutative. Two or more related results are also obtained.

KEY WORDS AND PHRASES. Commutative rings, torsion free rings, center of a ring, commutator ideal.

1980 AMS SUBJECT CLASSIFICATION CODES. 16A20, 116 A70.

1. INTRODUCTION.

Throughout this paper, $R$ will be an associative ring, $Z(R)$ the center of $R$, $N$ the set of all nilpotent elements of $R, N^{\prime}$ the set of all zero divisors of $R$, and $C(R)$ the commutator ideal of $R$. For any pair of elements $x, y$ in $R$, we set as usual $[x, y]=x y-y x$.

Recently, generalizing some results from Bell [1] Quadri and Khan [2,3]) proved that if $R$ is a ring satisfying the polynomial identity $\left[x y-y^{m} x^{m}, x\right]=0$ ( $m>1, n>1)$, then $R$ is commutative. In [4], Psomopoulos has shown that an s-unital ring $R$ in which the polynomial identity $\left[x^{n} y-y^{m} x, x\right]=0(m>1, n>0)$ holds, must be commutative.

In this paper, motivated by the above polynomial identities, we intend to prove results on commutativity of a ring $R$ with unity satisfying the fullowing property:

(i) "there exist positive integers $m>1$ and $n>1$ such that $\left[x^{n} y-y^{m} x^{n}, x\right]=0$ for al1 $x, y$ in $R^{\prime \prime}$.

Our property (i) can be regarded as an amalgam of those consider: 1 by the above authors. 


\section{PRELIMINARIES.}

In preparation for the proof of our results, we first state the following wellknown results.

LEMMA 2.1. (Psomopoulos [4]). Let $x, y \in R$. If $[x, y], x=0$, the for any positive integer $k,\left[x^{k}, y\right]=k x^{k-1}[x, y]$.

LEMMA 2.2. (Nicholson and Yaqub [5]). Let $R$ be a ring with unity 1 . Suppose that for some positive integer $k, x^{k} y=0=(x+1)^{k} y$ for all $x, y$ in $R$. The $y=0$.

LEMMA 2.3. (Bell [6]). Let $\mathrm{f}$ be a polynomial identity in a finite number of noncommuting indeterminates with integral coefficients. Then the following are equivalent:

(i) For any ring $R$ satisfying $f=0, C(R)$ is a nil ideal.

(ii) For every prime $p,(G F(p))_{2}$ fails to satisfy $f=0$.

LEMMA 2.3. (Tong [7]). Let $R$ be a ring with unity 1 . Let $I_{0}^{r}(x)=x^{r}$. If $k>1$, let $I_{k}^{r}(x)=I_{k-1}^{r}(x+1)-I_{k-1}^{r}(x)$. Then $I_{r-1}^{r}(x)=1 / 2(r-1) r$ ! $+r ! x ; I_{r}^{r}(x)=r$ ! , and $I_{j}^{r}(x)=0$ for $j>r$.

\section{RESULTS.}

Throughout the rest of the paper, $R$ stands for a ring with unity 1 , and satisfies the property (1). Let us first note that for any $x$, $y$ in $R$, the property (i) can also be expressed as:

$$
x^{n}[x, y]=\left[x, y^{m}\right] x^{n} \text {. }
$$

Then for any positive integer $t$, we obtain

$$
\begin{aligned}
& x^{t n}[x, y]=x^{(t-1) n}\left[x, y^{m}\right] x^{n}=x^{(t-2) n} \\
& {\left[x, y^{m^{2}}\right] x^{2 n}=x^{(t-3) n}\left[x, y^{m^{3}}\right] x^{3 n}=}
\end{aligned}
$$

By repeating the above process and using ( 3.1$)$, we get

$$
x^{t n}[x, y]=\left[x, y^{m^{t}}\right] x^{t n} \text {. }
$$

We also need the following two results for the proof of our main theorem.

LEMMA 3.1. Let $R$ be a ring with unity which satisfies the property (P). Then $N$ $Z(R)$.

PROOF. Let $u \in N$. Then by (3.2) for any $x \in R$ and a positive integer $t$, we have

$$
x^{t n}[x, u]=\left[x, u^{m}\right] x^{t n}
$$

But we have $u$ as a nilpotent element, then $u^{m^{t}}=0$, for sufficiently large $t$. Therefore, $x^{t n}[x, u]=0$ for all $x$ in $R$. Then we have $(x+1)^{t n}[x, u]=0$ for all $x$ in R. By Lemma 2.2, this implies that $[x, 11]=0$, which forces $N Z(R)$.

LEMMA 3.2. Let $R$ be a ring with unity which satisfies the property (i). Then $C(R) \quad Z(R)$. 
PROOF. Replacing $x$ by $(x+1)$ in (3.1) and inultiplying both sides by $x^{n}$ on the right and again using (3.1), we get

$$
(x+1)^{n}[x, y]^{n}=x^{n}[x, y](x+1)^{n} \text {, for } x, y \in R .
$$

Define

$$
E_{11}=\left(\begin{array}{ll}
1 & 0 \\
0 & 0
\end{array}\right), \quad E_{12}=\left(\begin{array}{ll}
0 & 1 \\
0 & 0
\end{array}\right) \text {, and } E_{21}=\left(\begin{array}{ll}
0 & 0 \\
1 & 0
\end{array}\right) \text {. }
$$

Let $x=E_{11}+E_{12}+E_{12}$ and $y=E_{11}$. Then $x$ and $y$ fail to satisfy (3.3) in $(G F(p))_{2}$, for a prime p. So by Lemma $2.3, C(R)$ is a nil ideal, and hence by Lemma 3.1 , $C(R) Z(R)$. This ends the proof.

In view of Lemma 3.2, it is guaranteed that the condition of Lemma 2.1 holds for each pair of elements $x, y$ in a ring $R$ with unity which satisfies the property (i).

THEOREM 3.1. Let $R$ be a ring with unity 1 satisfying property

(i) Then $R$ is commutative.

PROOF. We are given that $R$ is a ring with unity. So $R$ is isomorphic to a subdirect sum of subdirectly irreducible rings $R_{i}$ ( $i \varepsilon I$ ), each of which as a homomorphic image of $R$, satisfing the property (i). Thus we may assume that $R$ is a subdirectly irreducible ring satisfying (i).

Let $S$ be the intersection of all non-zero ideals of $R_{1}$. Then clearly $S \neq(0)$.

Now, if $n=1$ in the polynomial identity (3.1), we obtain $\left[x, y-y^{m}\right]=(x+1)[x, y]$ $-\left[x, y^{m}\right](x+1)-x[x, y]-\left[x, y^{m}\right] x=0$ for all $x, y \varepsilon R$. Thus $R$ is commutative by Herstein [8, Theorem 18].

Henceforth we assume $n>1$. Consider the positive integer $k=p^{m}-p$, where $p$ is a positive integer greater than 1 . Then by (3.1) we get

$$
\begin{aligned}
k x^{n}[x, y] & =p^{m} x^{n}[x, y]-p x^{n}[x, y] \\
& =p^{m}\left[x, y^{m}\right] x^{n}-p x^{n}[x, y] \\
& =\left[x,(p y)^{m}\right] x^{n}-x^{n}[x,(p y)] \\
& =x^{n}[x,(p y)]-x^{n}[x,(p y)]
\end{aligned}
$$

Thus $k x^{n}[x, y)=0$, whcih on replacing $x$ by $(x+1)$ yields $k[x, y]=0$. Now combining Lemma 3.2 with Lemma 2.1 , we have $\left[x^{k}, y\right]=k x^{k-1}[x, y]=0$. Therefore, it follows that

$$
x^{k} \varepsilon Z(R) \text { for all } x \text { in } R \text {. }
$$

Now, replacing $y$ by $y^{m}$ in $(3.1)$ we get

$$
x^{n}\left[x, y^{m}\right]=\left[x,\left(y^{m}\right)^{m}\right] x^{n} .
$$

Then by Lemma 3.2 , we have

$$
x^{n}\left[x, y^{m}\right]=\left[x, y^{m}\right] x^{n} .
$$

But

$$
\left[x, y^{m}\right]=m y^{m-1}[x, y]
$$


So using (3.6) and (3.7) we obtain

$$
x^{n}\left[x, y^{m}\right]=m y^{m-1}\left[x ; y^{m}\right] x^{n},
$$

and

$$
\begin{aligned}
{\left[x,\left(y^{m}\right)^{m}\right] x^{n} } & =m\left(y^{m}\right)^{m-1}\left[x, y^{m}\right] x^{n} \\
& =m y^{m-1} y^{(m-1)^{2}}\left[x, y^{m}\right] x^{n} .
\end{aligned}
$$

Thus (3.5) gives

$$
m y^{m-1}\left(1-y(m-1)^{2}\right) \quad\left[x, y^{m}\right] \quad x^{n}=0 .
$$

Let us replace $x$ by $(x+1)$ in $(3.8)$. Then we get $m y^{m-1}\left(1-y(m-1)^{2}\right)\left[x, y^{m}\right](x+1)^{n}=0$. So by Lemma $2.2, \mathrm{~m} \mathrm{y}^{\mathrm{m}-1}\left(1-\mathrm{y}^{(\mathrm{m}-1)^{2}}\right)\left[\mathrm{x}, \mathrm{y}^{\mathrm{m}}\right]=0$. Therefore, by Lemma 2.3 of Quadri and Khan [3], we have

$$
m y^{m-1}\left(1-y^{k(m-1)^{2}}\right)\left[x, y^{m}\right]=0 \text {. }
$$

Now, let $u \in N^{\prime}$. Then by (3.4), $u^{k(m-1)^{2}} \varepsilon N^{\prime} Z(R)$, and $s u^{k(m-1)^{2}}=(0)$. Hence using (3.9) we obtain

$$
m u^{m-1}\left[x, u^{m}\right]\left(1-u^{k(m-1)^{2}}\right)=0 \text {. }
$$

If $m u^{m-1}\left[x, u^{m}\right] \neq 0$, then $\left(1-u^{k(m-1)^{2}}\right) \in N^{\prime}$ and so $S=S\left(1-u^{k(m-1)^{2}}\right)=(0)$, which gives a contradiction as $S \neq(0)$. Therefore

$$
m u^{m-1}\left[x, u^{m}\right]=0 \text {. }
$$

Now, from (3.1) we have

$$
\begin{aligned}
x^{2 n}[x, u] & =\left[x, u^{m^{2}}\right] x^{2 n} \\
& =m u^{m(m-1)}\left[x, u^{m}\right] x^{2 n} \\
& =m u^{m-1} u^{(m-1)^{2}}\left[x, u^{m}\right] x^{2 n} \\
& =m u^{m-1}\left[x, u^{m}\right] u^{(m-1)^{2}} x^{2 n} .
\end{aligned}
$$

This implies that $x^{2 n}[x, u]=0$. Hence by Lemma 2.2 , we obtain $[x, u]=0$, that is

$u \in Z(R)$. Therefore, $N^{\prime} Z(R)$.

Clearly, for any $x \in R, x^{k}$ and $x^{k m} \varepsilon Z(R)$. Then by (3.1) for any $y \varepsilon R$, we have the identity

$$
\begin{aligned}
\left(x^{k}-x^{k m}\right) x^{n}[x, y] & =x^{k}\left(x^{n}[x, y]\right)-x^{k m}\left(x^{n}[x, y]\right) \\
& =x^{n}\left(x^{k}[x, y]\right)-\left(x^{k m}[x, y]\right) x^{n} \\
& =x^{n}\left[x, x^{k} y\right]-\left[x,\left(x^{k} y\right)^{m}\right] x^{n} \\
& =x^{n}\left[x, x^{k} y\right]-x^{n}\left[x, x^{k} y\right]
\end{aligned}
$$

Therefore,

$$
\begin{aligned}
& \left(x^{k}-x^{k m}\right) x^{n}[x, y]=0 \text {, and } \\
& \left(x-x^{t}\right) x^{s}[x, y]=0,
\end{aligned}
$$

where $t=k m-k+1$, and $s=n+k-1$. 
Now, if $x^{s}[x, y]=0$, then Lemma 2.2 yields $[x, y]=0$. But $x^{s}[x, y] \neq 0$ gives $x-x^{t} \in N^{\prime} \quad Z(R)$.

Therefore, $\left[x-x^{t}, y\right]=0$, for all $x, y$ in $R$, which implies that $[x, y]=0$, by Theorem 18 of [9]. Thus in evey case $[x, y]=0$.

This proves that $R$ is commutative.

THEOREM 3.2. Let $R$ be an s-unital ring satisfying the property ( 1 ). Then $R$ is commutative.

PROOF. This follows from Proposition 1 of Hirano, Kobayashi and Tominaga [9] since $R$ with unity satisfying (i) is commutative by Theorem 3.1. Finally, we present a short and easy proof of Theorem 3.1, but under an extra condition on the commutators in the ring $R$. We use an iteration technique as given in Tong [7].

THEOREM 3.3. Let $R$ be a ring with unity satisfying the property (i). If every commutator in $R$ is $m$ ! - torsion free, then $R$ must be commutative.

PROOF. The ring $R$ satisfies the identity

$$
\left.x^{n}[x, y]=\left[x, y^{m}\right] x^{n}, n>1, m>1\right) .
$$

We shall apply the iteration on $y^{m}$. As in $[7]$, let $I_{j}(y)=I_{j}^{m}(y)$ for $j=0,1,2, \ldots$. Then the above identity can be rewritten as

$$
x^{n}[x, y]=\left[x, I_{0}(y)\right] x^{n}
$$

Replacing $y$ by $(y+1)$ in $(3.11)$, we obtain

$$
x^{n}[x, y]=\left[x, I_{0}(y+1)\right] x^{n} .
$$

Now, using Lemma 2.4, we get

$$
x^{n}[x, y]=\left[x, I_{0}(y)+I_{1}(y)\right] x^{n} .
$$

Equations (3.11) and (3.12) when combined, give

$$
0=\left[x, I_{1}(y)\right] x^{n} \text {. }
$$

Again, let $y=y+1$ in (3.13). Then using Lemma 2.4 we have

$$
0=\left[x, I_{2}(y)\right] x^{n} \text {. }
$$

Repeating the above process $(m-1)$ times, we reach the identity

$$
0=\left[x, I_{m-1}(y)\right] x^{n} \text {. }
$$

With an application of Lemma 2.4 , we end up with

$$
\mathrm{m} ![\mathrm{x}, \mathrm{y}] \mathrm{x}^{\mathrm{n}}=0 \text {. }
$$

Now replacing $x$ by $(x+1)$ in the above identity, and making ue of Lemma 2.2 , we have

$$
m ![x, y]=0 \text {, for all } x, y \in R \text {. }
$$

By every commutator in $R$ is $m$ !-torsion free, so $[x, y]=0$ for all $x$ and $y$ in $R$. Therefore, $R$ is commutative. This completes the proof. 


\section{REFERENCES}

1. BELL, H.E., Some commutativity results for rings with two variable constraints, Proc. Amer. Math. Soc. 53 (1975), 280-285.

2. QUADRI, M.A. and KHAN, M.A., A commutativity theorem for associative rings, Math. Japon 33(2) (1988), 275-279.

3. QUADRI, M.A. and KHAN, M.A., A commutativity theorem for left s-unital rings, Bu11. Inst. Math. Acad. Sinica, 15(3) (1987), 323-327.

4. PSOMOPOULOS, E., A commutativity theorem for rings, Math. Japon 29 (1984), 371373.

5. NICHOLSON, W.K. and YAQUB, A., A commutativity theorem for rings and groups, Canad. Math. Bul1. 22 (1979), 419-423.

6. BELL, H.E., On some commutativity theorems of Herstein, Arch. Math. 24 (1973), 3438.

7. TONG, J., On the commutativity of a ring with identity, Canad. Math. Bull. 27(4) (1984), 456-460.

8. HERSTEIN, I.N., A generalization of a theorem of Jacobson, Amer. J. Math. 73 $(1951), 756-762$.

9. HIRANO, Y., KOBAYASHI, Y. and TOMINAGA, H., Some polynomial identities and commutativity of s-unital rings, Math. J. Okayama Univ. 24 (1982), 7-13. 


\section{Journal of Applied Mathematics and Decision Sciences}

\section{Special Issue on}

\section{Intelligent Computational Methods for Financial Engineering}

\section{Call for Papers}

As a multidisciplinary field, financial engineering is becoming increasingly important in today's economic and financial world, especially in areas such as portfolio management, asset valuation and prediction, fraud detection, and credit risk management. For example, in a credit risk context, the recently approved Basel II guidelines advise financial institutions to build comprehensible credit risk models in order to optimize their capital allocation policy. Computational methods are being intensively studied and applied to improve the quality of the financial decisions that need to be made. Until now, computational methods and models are central to the analysis of economic and financial decisions.

However, more and more researchers have found that the financial environment is not ruled by mathematical distributions or statistical models. In such situations, some attempts have also been made to develop financial engineering models using intelligent computing approaches. For example, an artificial neural network (ANN) is a nonparametric estimation technique which does not make any distributional assumptions regarding the underlying asset. Instead, ANN approach develops a model using sets of unknown parameters and lets the optimization routine seek the best fitting parameters to obtain the desired results. The main aim of this special issue is not to merely illustrate the superior performance of a new intelligent computational method, but also to demonstrate how it can be used effectively in a financial engineering environment to improve and facilitate financial decision making. In this sense, the submissions should especially address how the results of estimated computational models (e.g., ANN, support vector machines, evolutionary algorithm, and fuzzy models) can be used to develop intelligent, easy-to-use, and/or comprehensible computational systems (e.g., decision support systems, agent-based system, and web-based systems)

This special issue will include (but not be limited to) the following topics:

- Computational methods: artificial intelligence, neural networks, evolutionary algorithms, fuzzy inference, hybrid learning, ensemble learning, cooperative learning, multiagent learning
- Application fields: asset valuation and prediction, asset allocation and portfolio selection, bankruptcy prediction, fraud detection, credit risk management

- Implementation aspects: decision support systems, expert systems, information systems, intelligent agents, web service, monitoring, deployment, implementation

Authors should follow the Journal of Applied Mathematics and Decision Sciences manuscript format described at the journal site http://www.hindawi.com/journals/jamds/. Prospective authors should submit an electronic copy of their complete manuscript through the journal Manuscript Tracking System at http://mts.hindawi.com/, according to the following timetable:

\begin{tabular}{|l|l|}
\hline Manuscript Due & December 1, 2008 \\
\hline First Round of Reviews & March 1,2009 \\
\hline Publication Date & June 1, 2009 \\
\hline
\end{tabular}

\section{Guest Editors}

Lean Yu, Academy of Mathematics and Systems Science, Chinese Academy of Sciences, Beijing 100190, China; Department of Management Sciences, City University of Hong Kong, Tat Chee Avenue, Kowloon, Hong Kong; yulean@amss.ac.cn

Shouyang Wang, Academy of Mathematics and Systems Science, Chinese Academy of Sciences, Beijing 100190, China; sywang@amss.ac.cn

K. K. Lai, Department of Management Sciences, City University of Hong Kong, Tat Chee Avenue, Kowloon, Hong Kong; mskklai@cityu.edu.hk 